\title{
Error Analysis of the Numerical Method for Correcting the Propagation of EM waves in the Troposphere
}

\author{
Yuanzhi Liu'1,2,4(D), Tao Tang 2,3,5(D), Guo Liu'1(D), M. Olaimat ${ }^{3}$ (D) \\ ${ }^{I}$ Science and Technology on Electronic Information Control Laboratory, Southwest China Research Institute \\ of Electronic Equipment, Chengdu 610036, China, \\ ${ }^{2}$ College of Electronic Engineering, Chengdu University of Information Technology, Chengdu 610025, China, \\ ${ }^{3}$ Department of Electrical and Computer Engineering, University of Waterloo, Waterloo N2L 3G1, Canada, \\ ${ }^{4}$ School of Electrical Engineering and Computer Science, University of Ottawa, Ottawa K1N 6N5, Canada, \\ ${ }^{5}$ Key laboratory of Atmospheric Sounding, China Meteorological Administration, Chengdu 610225, China, \\ yliu600@uottawa.ca,tangt@cuit.edu.cn,liuguosgg@163.com,olaimat_melad@yahoo.com
}

\begin{abstract}
Electromagnetic (EM) waves do not travel in straight lines in the troposphere due to refraction. This leads to errors in many engineering applications. In order to correct these errors, a numerical solution based on the difference method has been proposed, which can reveal the degree of EM wave bending owing to atmospheric refraction, moreover, it can predict the actual path of the EM wave propagating in the troposphere. The error of the numerical method is studied here, which reveals the source of the error. Based on this, two improvement methods are proposed. The computational accuracy of the numerical methods is verified by comparison with the analytical solution. The proposed method ensures the computational efficiency and accuracy of the problem of refraction of EM waves in the troposphere.
\end{abstract}

Index Terms - Difference method, propagation path, troposphere refraction, error analysis.

\section{INTRODUCTION}

Electromagnetic (EM) waves propagate in straight lines in uniform media. However, the troposphere is a non-uniform medium in which the refractive index varies with height. Consequently, when an EM wave, such as a radio wave, emits diagonally into the atmosphere, it will be bent, and its propagation path will be deflected away from a straight line. In some applications, such as: radar detection, space wireless communication, and other electromagnetic energy transforms, the locations of the concerned objects need to be modified by appropriate correction methods, owing to the significant errors that are caused by wave refraction. Previously, the propagation process of EM waves in the troposphere has been modeled [1], [2], and some measurement methods of atmospheric effects on EM waves have been presented [3]-[6]. Several methods, that focus on the refraction phenomenon of the EM wave in the atmosphere, have been proposed as well [7]-[9].

To track the propagation path of EM wave in the troposphere, analytical solutions with pure mathematics are usually used [10]-[12]. However, these methods often suffer from issues such as: complexity, inefficiency and inaccuracy. For example, a tropospheric range-error correction method, 
based on a stratified refractive index to calculate the range-error of the path of EM waves, was deduced with a standard error of $8 \%$ [7].

If the propagation path of EM waves can be identified and demonstrated by figures, one can deal with the mentioned errors. Based on this idea, a numerical method has been presented to identify the offset error, and to track the EM wave's propagation path in the troposphere [13]. The proposed numerical solution is based on the difference method. By dividing the propagation path into small grids, the state of the EM waves at the next space can be deduced by the state of the EM waves at the front space, and thus the whole process of EM waves' propagation in the troposphere can be confirmed. Compared with the ray-tracing method [14], which is known as one of the mathematics solution, the proposed numerical method is much more simple. And it is often more convenient to represent radiation as rays of energy instead of waves [15], so, compared with the parabolic equation method [16], this method is only used to quickly estimate the propagation path of EM waves in the troposphere, but it cannot provide the distributions of the EM wave in the whole propagation spatial.

However, as a numerical solution, this method introduces its own errors. In this paper, the errors of the numerical method are analyzed and improvements are proposed on this basis. The computational accuracy of the improved method is verified by comparison with the analytical solution. The rest of the paper is organized as follows: Sec. 2 presents the physical model of the EM wave propagation process; Sec. 3 gives the simulation process of the numerical method; Errors analysis and an improvement method is proposed in Sec. 4, and Sec. 5 provides a conclusion.

\section{PhysicAl MODEL OF PROPAGATION PROCESS}

When EM waves propagate in a medium, its velocity is given by $v=c / n$, where $c$ is the speed of light in vacuum, and $n$ is the refractive index of the medium. When an EM wave emits into the troposphere with an elevation angle of $\theta$, the velocity of the wave can be decomposed into $v_{x}$ and $v_{y}$, which represent the velocity components in the horizontal and vertical directions, respectively, as shown in Fig. 1 (a). The refraction index of the troposphere, generally, remains constant over a wide transverse range, varying only with the height [1]. Therefore, $v_{x}$ and $v_{y}$ can be written as:

$$
v_{x}=c \cdot \cos \theta, \quad v_{y}=c / n(h) \cdot \sin \theta
$$

Set $\varphi$ as the geocentric offset angle, the wave will be shifted from its initial position over time $t$. If $\varphi$ is small enough, in polar coordinates, the following approximate equations can be obtained:

$$
v_{x} t=R \cdot \varphi, \quad v_{y} t=h
$$

where $R$ is the distance from the emission site to the center of the earth, which is the altitude of the EM source plus the earth radius, and $h$ is the height increasing in vertical direction within time $t$ of the wave. Therefore, the following equation can be obtained from (1) and (2):

$$
h=\frac{R \cdot \varphi}{n(h)} \cdot \tan \theta
$$


From (3), if the values of $h$ and $\varphi$ are obtained, the trajectory of the EM wave then can be determined. But in (3), only $R$ and $\theta$ are known, and $n(h)$ varies with height. So, (3) is an implicit function, and cannot be solved directly.
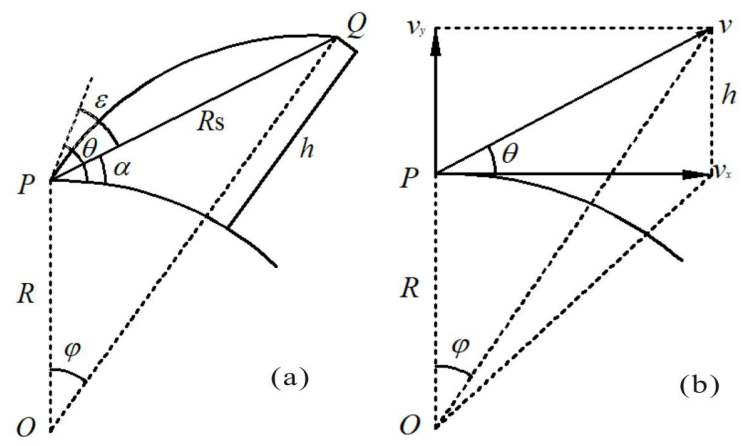

Fig. 1: The schematic of an EM wave that propagates in the troposphere. (a) EM wave in radar detection, (b) the velocity of the EM wave.

\section{THE NUMERICAL METHOD}

As mentioned previously, the refractive index of the troposphere is a function of altitude, and the range of change is very small (refractivity index $N$ of the troposphere is defined by refractive $n$ as: $\left.N=(n-1) \times 10^{6}\right)$ [12]. Therefore, troposphere can be considered as a superposition of layers of different mediums with different refractive indexes. If an EM wave is incident into the troposphere with a certain initial elevation angle and altitude, then we can set the following parameters for computing: $d \varphi$ is the deviation of the geocentric offset angle from the original position after a period of time $d t$ in each layer, and $d h$ is the height increment in the vertical direction during the period of $d t$. Besides, since $d t$ is very small, the corresponding $d h$ is also very small, so, the refractive index of the troposphere in each $d h$ can be regarded as a constant.

Because $d \varphi$ is very small, (3) can be applied separately to the propagation of the EM waves in each layer, and the path of the wave in the troposphere can be determined by summing up the propagation trajectory of the waves in each layer. The whole process can be illustrated by Fig. 2. In Fig. 2, point $O$ is the center of the earth, point $A$ is the location of the EM source. The whole propagation process can be concluded as follows:

Stage 1: the refractive index at the emission point is determined to be $n_{1}$. The initial emission elevation angle $\theta_{1}$ is known, set $d \varphi$ to be a very small value such as $1 / 100$ degree. The propagation distance in the vertical direction of the wave $d h_{k}$ can be solved by the following equation:

$$
d h_{k}=\frac{R_{k} \cdot d \varphi}{n_{k}} \cdot \tan \theta_{k}
$$

At stage $1, R_{1}$ is the distance from the EM source to the center of the earth, which is a known parameter. For a given atmospheric refraction model, $n_{1}$ is a constant as well. So, $d h_{1}$ can be obtained. 
Stage 2: when the EM wave travels to a new location, which is considered as the new emission site, the distance from this location to the center of the earth is:



Fig. 2. Schematic diagram of tropospheric layering in EM wave propagation.

$$
R_{k}=R_{k-1}+d h_{k-1} \quad(k=2,3,4 \mathrm{~L})
$$

At this stage, $k=2$. The new emission elevation $\theta_{2}$ can be obtained by using the Snell law on the interface between the first and second layer. In the triangle $A O B$ of Fig. 2, the angle of incidence can be determined as $\left[\pi-\left(\pi / 2+\theta_{1}+d \varphi\right)\right]$ and the angle of refraction is $\left(\pi / 2-\theta_{2}\right)$, so the following equation can be obtained:

$$
\frac{\sin \left[\pi-\left(\pi / 2+\theta_{1}+d \varphi\right)\right]}{\sin \left(\pi / 2-\theta_{2}\right)}=\frac{n_{2}}{n_{1}}
$$

For a selected refractive index model, the refractive index $n_{2}$ can also be calculated directly. Using (4), (5) and (6), $d h_{2}$ can be solved.

Every stage can be calculated in the same way. The whole increasing height of the wave in the vertical direction is $h=\sum_{k=1}^{k} d h_{k}$, the whole geocentric offset angle $\varphi=k \cdot d \varphi$. So, the whole path of the EM wave propagation in the troposphere can be determined.

In Fig. 1 (a), point $P$ is the source site of the EM wave, point $Q$ is the actual location of the end point of the EM wave after a period of time. So, the arc of Fig. 1 (a) is the actual path of the radio wave. $\theta$ is the initial emission elevation angle, $\alpha$ is the elevation angle of connection between the source point and the real propagation end point, that is the elevation angle of straight line $P Q$ which is smaller than $\theta, \varepsilon$ is the elevation angle error between linear propagation and real propagation, which can be given by:

$$
\varepsilon=\theta-\alpha
$$




\section{RESULTS AND ERROR ANALYSIS}

The feasibility and accuracy of the proposed numerical method can be verified by analytic solution results. The refractive index of troposphere in Beijing is given by [17]:

$$
N(h)= \begin{cases}320-41\left(h-h_{s}\right) & h_{s} \leq h \leq h_{s}+1 \mathrm{~km} \\ 279 e^{-0.1225\left(h-h_{s}-1\right)} & h_{s}+1 \mathrm{~km}<h \leq 9 \mathrm{~km} \\ 105 e^{-0.1434(h-9)} & 9 \mathrm{~km}<h \leq 60 \mathrm{~km}\end{cases}
$$

where $h_{s}$ is the altitude of Beijing, which is $0.333 \mathrm{~km}$. When the EM source placed on the surface of the earth, the distance $R_{1}$ will be $R_{1}=6371 \mathrm{~km}+h_{s}$. In the triangle $P O Q$ of Fig. 1 (a), according to the sine theorem, the value of elevation angle of $P Q$ can be calculated by the following equation:

$$
\frac{\sin (\pi / 2+\alpha)}{h+R}=\frac{\sin \varphi}{R_{s}}
$$

From (7), the elevation angle error of EM waves propagating in the troposphere of Beijing can be obtained. Ref [13] has given the verification process for the analytical solution. The angle error can also be obtained by substituting the Beijing refractive index into the analytical solution.

Fig. 3 compares relation between the increased height $h$ and the elevation angle error $\varepsilon$ that was obtained by the proposed numerical method and the analytical solution. As illustrated in Fig. 3, the simulation results are almost identical to the analytical solutions. But this method has errors of about $2.9 \%$.

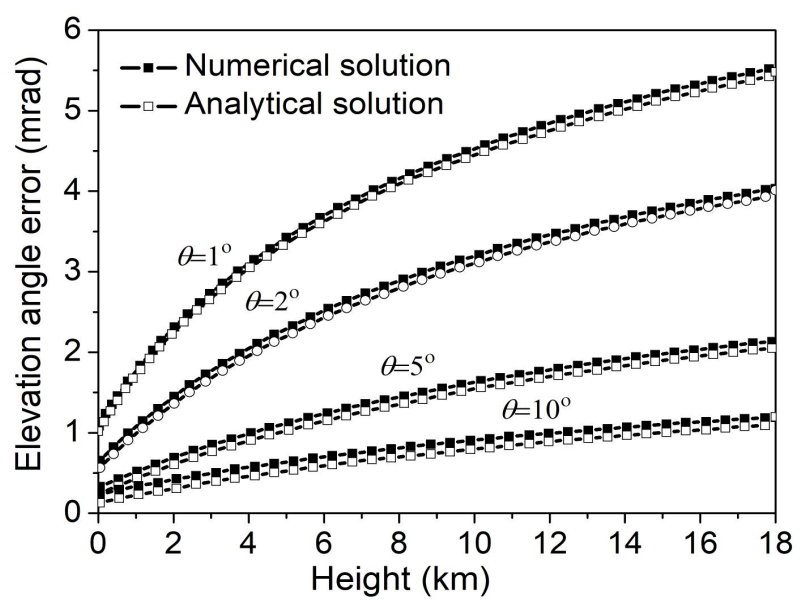

Fig. 3. The relation between elevation angle error and height obtained by the numerical method and the analytical solution.

It is obvious that when $n=1$, the path of the wave must be a straight line and the elevation angle error must be 0 . Set $n=1$ in the proposed numerical method, the elevation angle errors obtained are presented in Fig. 4 (a). According to Fig. 4 (a), the results obtained by the proposed method have some errors. For example, there is an error of $0.0873 \mathrm{mrad}$, approximately, when $\theta=1^{\circ}$.

In the proposed numerical method, we keep the $d \varphi$ as a constant, to calculate the $d h_{k}$ in each layer. But the refractive index is decreasing with the increasing altitude. So, the incident angle of each layer will be smaller and smaller, consequently, $d h_{k}$ will be lager and lager. After a long propagation 
distance, $d h_{k}$ may become so large introducing errors in the case where the refractive index is no longer constant in each $d h_{k}$.

If we keep $d h$ as a constant, form (4) we have:

$$
d \varphi_{k}=\frac{n_{k} \cdot d h}{R_{k} \cdot \tan \theta_{k}}
$$

So, the whole path of the EM wave propagation in the troposphere can be determined by $\varphi=\sum_{k=1}^{k} d \varphi_{k}$ and $h=k \cdot d h$. As long as $d h$ is small enough to satisfy the condition of the refractive index being constant for each layer, the error introduced by the differential method can be reduced.

Besides, the errors shown in Fig. 4 (a) can be noted as the systematic error caused by the numerical method, which can be defined as $o$. After it was introduced, (7) can be modified to:

$$
\varepsilon=\theta-\alpha-o
$$

Substituting the values obtained in Fig. 4 (a) into (11) as the values of $o$, Fig. 4 (b) shows the revised results. From Fig. 4 (b), we can get that the results of the two improved methods are in high agreement with those of the analytical solution. It fully meets the requirements for calculation accuracy.
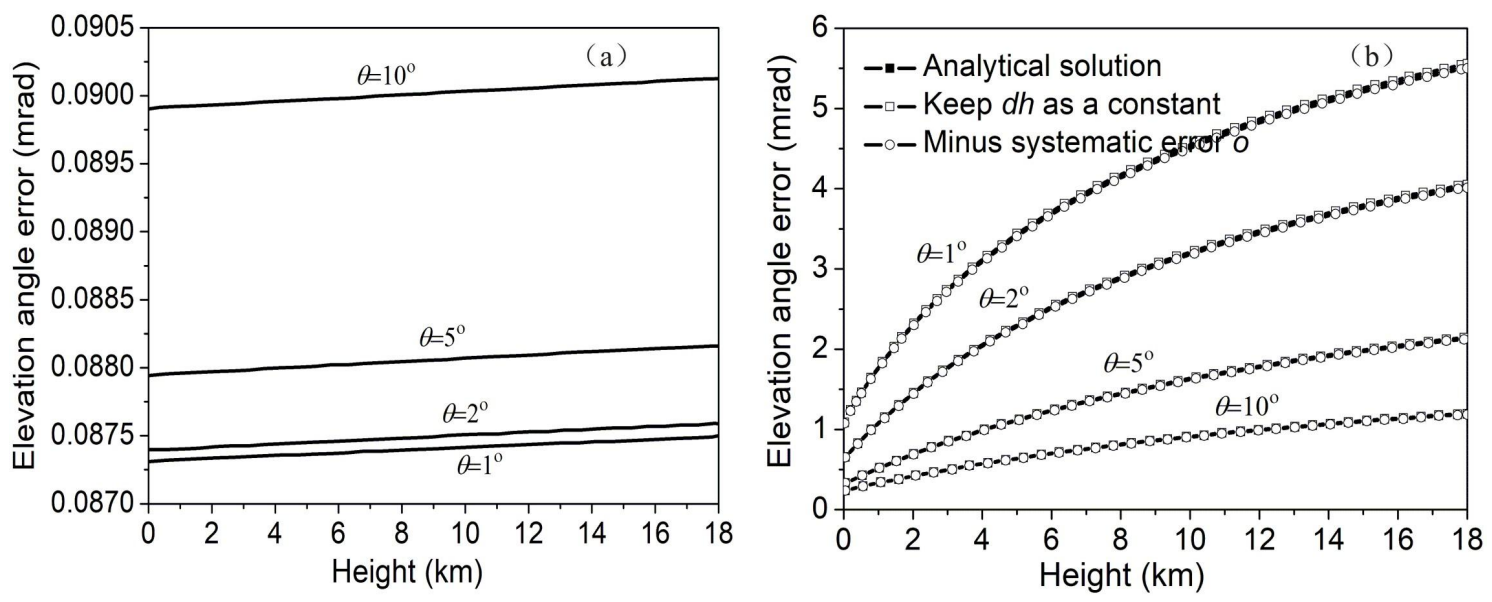

Fig. 4. Angle errors. (a) the elevation angle errors calculated by the numerical method when $n=1$. (b) the modified relation between elevation angle error and height.

As the whole propagation distance in the vertical direction $h$ and the whole offset angle $\varphi$ of the EM wave have been obtained, the propagation path can be plotted. Fig. 5 shows the trajectory of the EM wave whose initial elevation angle is $1^{\circ}$ with refraction given by (8) and without refraction $(n=1)$, respectively (note that the scale of horizontal and vertical coordinates is inconsistent).

From Fig. 5, it can be seen that the refraction obviously causes the deviation of the path. At the beginning, the path is apparently an arc which is caused by the refractive index calculated from (8). However, the refractive index decreases and finally tends to about 1 as the height increases, and the influence caused by the change of refractive index also becomes smaller. 


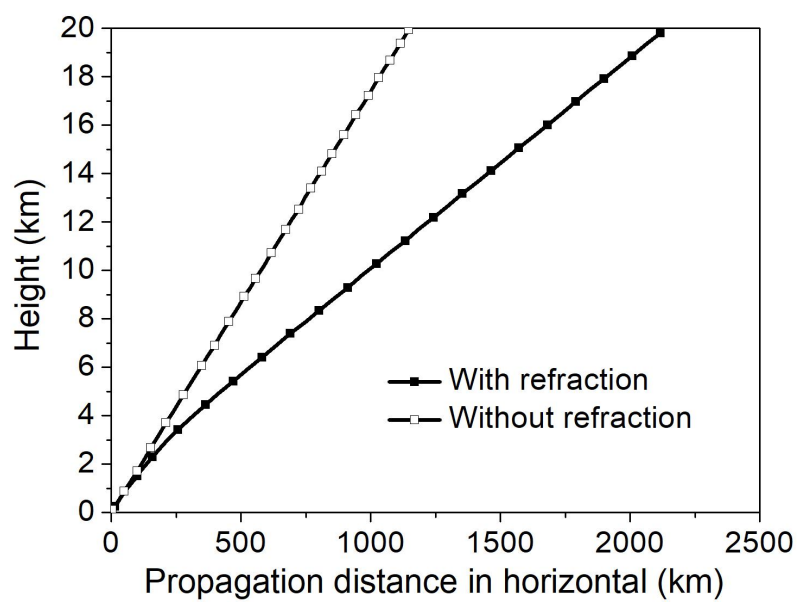

Fig. 5. The propagation path of EM waves with and without refraction respectively.

\section{CONCLUSION}

By dividing the troposphere into layers, and combing with the Snell refraction law, the propagation path of EM waves can be determined by numerical solution methods. The calculation process of the numerical method which is based on the idea of difference is presented. Error analysis of the proposed numerical solution is also discussed, and two improvement methods were presented. The improvement methods introduced guarantee the feasibility of the numerical method; comparison results with the analytical solution demonstrates the high accuracy of this modification method. The proposed numerical method can be utilized to quickly estimate atmospheric refraction errors and can applied to different areas, with different refractive index model.

\section{ACKNOWLEDGMENT}

This work was supported in part by National Natural Science Foundation of China (No. 61701082, No. 61701116, No. 61601093, No. 61971113 and No. 61901095) in part by National Key R\&D Program project (No. 2018YFB1802102, No. 2018AAA0103203), in part by National Study Abroad Fund of China (No. 201808515077), in part by Guangdong Provincial Research and Development Plan in Key Areas (No. 2019B010141001, No. 2019B010142001), in part by Sichuan Provincial Science and Technology Planning Program (No. 2018HH0034, No. 2019YFG0418, No. 2019YFG0120, No. 2018JY0246), in part by the Fundamental Research Funds for the Central Universities (No. ZYGX2016J004), and in part by Science and Technology on Electronic Information Control Laboratory (No. HX1122).

\section{REFERENCES}

[1] Y. Yang, Y. Long, "Modeling EM pulse propagation in the troposphere based on the TDPE method," IEEE Antennas and Wireless Propagation Letters, vol. 12, no. 2, pp. 190-193, Jan. 2013.

[2] A. Popov, V. Kopeikin and F. M. Landstorfer, "Full-wave simulation of overland radar pulse propagation," Electronics Letters, vol. 39, no. 6, pp. 550-552, Mar. 2003.

[3] W. Panpan, Z. Chen and Z. Zhengyu, "Numerical study of radio wave propagation in clear air acoustic scatterer," in Journal of Systems Engineering and Electronics, vol. 29, no. 4, pp. 684-692, Aug. 2018.

[4] E. A. Karagianni, A. P. Mitropoulos, I. Latif, A. Kavousanos-Kavousanakis, J. Koukos, and M. E. Fafalios, "Atmospheric effects on em propagation and weather effects on the performance of a dual band antenna for wlan communications," Nausivios Chora, vol. 5, pp.B29-B45, 2014. 
[5] V. B. Mendes, G. Prates, E. C. Pavlis, D. E. Pavlis, and R. B. Langley, "Improved mapping functions for atmospheric refraction correction in SLR," Geophysical Research Letters, vol. 29, no. 10, pp. 531-534, May 2002.

[6] M. Zhu, and J. Liu, "The design of microwave resonator to accurately measure the atmospheric refractivity," Physics Procedia, vol. 33, pp. 1670-1676, 2012.

[7] H. D. Black, A. Eisner, "Correcting satellite Doppler data for tropospheric effects," Journal of Geophysical Research, vol. 89, no. D2, pp. 2616-2626, Apr. 1984.

[8] B. Chen , Z. Liu, "A comprehensive evaluation and analysis of the performance of multiple tropospheric models in China region," IEEE transactions on geoscience and remote sensing, vol. 54, no. 2, pp. 663-678, Feb. 2016.

[9] I. M. Ifadis, "The delay mismodelling due to pointing error in precise space to earth geodetic observations," Survey Review, vol. 36, no.282, pp. 273-281, Jul. 2013.

[10] B. Goudar, R. J. Watson, "Variability in propagation path delay for atmospheric remote sensing," in Proc. 2014 IEEE Geoscience and Remote Sensing Symposium, Quebec City, QC, Canada, 2014, pp. 4127-4130.

[11] G. D. Thayer, "A rapid and accurate ray tracing algorithm for a horizontally stratified atmosphere," Radio Science, vol. 2, no. 2, pp. 249-252, Feb. 1967.

[12] C. Levis, J. T. Johnson, F. L. Teixeira, "Radiowave propagation: physics and applications," in Hoboken, New Jersey US: John Wiley \& Sons, 2010, pp. 120-134.

[13] T. Tang, G. Liu, L. Liu, "Numerical modeling the propagation path of radio waves with atmospheric refractivity," Microwave and Optical Technology Letters, vol. 62, pp. 1651- 1655, Dec. 2019.

[14] Z. Yun, M. F. Iskander, "Ray tracing for radio propagation modeling: Principles and applications," IEEE Access, vol. 3, pp. 1089-1100, Jul. 2015.

[15] E. A. Karagianni, A. P. Mitropoulos, I. Latif, A. Kavousanos-Kavousanakis, J. Koukos, and M. E. Fafalios, "Atmospheric effects on em propagation and weather effects on the performance of a dual band antenna for wlan communications" Nausivios Chora vol. 5, pp. B29-B45, 2014.

[16] K. H. Craig, "Propagation modelling in the troposphere: parabolic equation method," Electronics Letters, vol. 24, no. 18, pp. 1136-1139, Sept. 1988.

[17] J. Mo, R. Zhong, "A Method for Radio Refraction Error Correction of Troposphere," Fire Control and Command Control, vol. S1, pp. 164-165, 2010. (in Chinese). 